\title{
Potential Type Operators \\ on \\ Curves with Vorticity Points
}

\author{
V. Rabinovich
}

\begin{abstract}
We study potential type operators on certain non-Lipschitz curves $\Gamma$. The curves under consideration are locally Lyapunov except for a finite set $F$ of singular points. The normal vector $\nu(y)$ to the curve $\Gamma$ does not have a limit at the singular points and, moreover, $\nu(y)$ may be an oscillating and rotating vector function in a neighborhood of the singular points. We establish a Fredholm theory of potential type operators in the spaces $L_{p, w}\left(\Gamma, \mathbb{C}^{n}\right)$ where $p \in(1, \infty)$ and $w$ is a weight satisfying the Muckenhoupt condition.
\end{abstract}

Keywords: Potential operators, Fredholmness, essential spectrum

AMS subject classification: 31 A 10

\section{Introduction}

Let $\Gamma$ be a curve which is locally Lyapunov except for a finite set $F$ of points on $\Gamma$. We refer to the points in $F$ as the singular points of $\Gamma$. We consider operators of the form

$$
B u(x)=a(x) u(x)+\int_{\Gamma} \frac{(\nu(y), x-y)}{|x-y|^{2}} g(x, y) u(y) d l_{y} \quad(x \in \Gamma)
$$

where $d l_{y}$ is the oriented Lebesgue measure on $\Gamma, a$ and $g$ are bounded matrix functions on $\Gamma$ with elements having second kind discontinuities with respect to $x$ and $y$ at singular points of $\Gamma$, and $\nu(y)$ is the interior normal vector of the curve $\Gamma$ at the point $y \in \Gamma \backslash F$. We assume that the vector $\nu(y)$ does not approach a limit at singular points. Moreover, we allow $\nu(y)$ to be an oscillating and rotating vector function in a neighborhood of each singular point. Singular points of the latter kind will be called vorticity points. We require that the curves under consideration satisfy the well-known Carleson condition (see, for instance, [1]). The class of operators (1) includes the operators of harmonic potentials, wave potentials, and other operators on curves with vorticity points which are important in mathematical physics. The results of this paper allow us to consider interior and exterior boundary value problems in planar domains whose boundary has vorticity points.

V. Rabinovich: ESIME-Zacatenco, Nat. Polit. Inst., Dept Telecommun., Ed. 1, Av. IPN, Mexico 07738, D.F.MEXICO; rabinov@maya.esimez.ipn.mx; partially supported by Russian Fund of Fundamental Investigations, grant RFFI-98-01-01-023

ISSN 0232-2064 / \$2.50 C Heldermann Verlag Berlin 
We construct a Fredholm theory of operators (1) in the Lebesgue spaces $L_{p, w}\left(\Gamma, \mathbb{C}^{n}\right)$ with $p \in(1, \infty)$ and a weight $w$ satisfying the Muckenhoupt condition [1].

In the case where $\Gamma$ is a so-called Radon curve without peaks and $a$ and $b$ are piecewise continuous functions, potential operators have been considered by many authors, starting with Radon's classical paper [2]. Later these operators have been investigated by Y. B. Lopatinskiy [3], I. Daniluk [4], V. Shelepov [5], and other authors (see, for instance, the surveys $[6,7])$. Potential operators on curves with peaks are considered in papers by V. Maz'ya and A. Solov'ev (see, for example, [8 - 10]). Note that the class of curves studied here is not contained in the classes of curves treated in the aforementioned papers. It should be noted that the methods of the papers [ $3-5]$ are based on the theory of Wiener-Hopf equations on the half-line developed in the well-known paper of I. Gohberg and M. Krein [11]. But in the case of vorticity singular points this method is not applicable. Our approach is based on the theory of Mellin pseudodifferential operators. Earlier this approach was successfully applied to singular integral operators on some Carleson curves in the author's papers [12 - 14] and in joint work of the author with A. Böttcher and Yu. I. Karlovich [15 - 16].

The theory of potential type operators on curves with vorticity points differs essentially from the theory of such operators on "nice" curves. For instance, the operator

$$
A u(x)=\int_{\Gamma} \frac{(\nu(y), x-y)}{|x-y|^{2}} u(y) d l_{y} \quad(x \in \Gamma)
$$

where

$$
\Gamma=\left\{x \in \mathbb{C}: x=t \exp i \delta \ln t \text { with } t \in\left(0, t_{0}\right) \text { and } \delta \in \mathbb{R}\right\}
$$

is a piece of a logarithmic spiral, is not a compact operator in the space $L_{p}(\Gamma)$, which is in contrast to the case when $\Gamma$ is a simple Lyapunov arc of finite length. It should also be noted that oscillations of the curve $\Gamma$ and of the weight $w$ may lead to the appearance of massive local and Fredholm spectra of the operator $A: L_{p}(\Gamma, w) \rightarrow L_{p}(\Gamma, w)$.

The paper contains five sections. In Section 2 we provide some auxiliary material on Mellin pseudodifferential operators following the papers [13, 14, 17]. In Section 3 we consider potential type operators on certain simple unbounded curves $\gamma$. We construct a local symbol at singular points, give a criterion for Fredholmness and an index formula. We also prove a criterion for the compactness of certain operators in the spaces $L_{p, w}\left(\Gamma, \mathbb{C}^{n}\right)$. Section 4 is devoted to potential operators on closed Jordan curves with vorticity points. We give a description of the local and essential spectra and derive an index formula. In Section 5 we discuss applications of the results of Section 4 to the double layer potential operator for the interior Dirichlet problem. 


\section{Mellin pseudodifferential operators}

2.1 Main properties of Mellin pseudodifferential operators. We say that a matrix function $\left(a_{i j}(r, \lambda)\right)_{i, j=1}^{n}$ defined on $\mathbb{R}_{+} \times \mathbb{R}$ belongs to $\mathcal{E}^{m}(n)$ if the functions $a_{i j}(r, \lambda)$ are in $C^{\infty}\left(\mathbb{R}_{+} \times \mathbb{R}\right)$ and satisfy the estimate

$$
\sup _{\mathbb{R}_{+} \times \mathbb{R}}\left|\left(r \partial_{r}\right)^{\alpha} \partial_{\lambda}^{\beta} a_{i j}(r, \lambda)\right|(\lambda)^{-m+\beta}<\infty
$$

for all $\alpha, \beta \in \mathbb{N}_{0}=\{0\} \cup \mathbb{N}$, where $(\lambda\rangle=\left(1+\lambda^{2}\right)^{\frac{1}{2}}$. A matrix function $a(r, \lambda)\left(\in \mathcal{E}^{0}(n)\right)$ is said to be slowly varying at the point 0 if

$$
\lim _{r \rightarrow+0} \sup _{\lambda \in \mathbb{R}}\left|\left(r \partial_{r}\right)^{\alpha} \partial_{\lambda}^{\beta} a_{i j}(r, \lambda)\right|\langle\lambda\rangle^{\beta}=0
$$

for all $\alpha \in \mathbb{N}_{0}$ and $\beta \in \mathbb{N}$, and it is said to be slowly varying at the point $+\infty$ if

$$
\lim _{r \rightarrow+\infty} \sup _{\lambda \in \mathbb{R}}\left|\left(r \partial_{r}\right)^{\alpha} \partial_{\lambda}^{\beta} a_{i j}(r, \lambda)\right|(\lambda)^{\beta}=0
$$

for all $\alpha \in \mathbb{N}_{0}$ and $\beta \in \mathbb{N}$. We denote by $\widetilde{\mathcal{E}}(n)$ the class of matrix functions in $\mathcal{E}^{0}(n)$ which are slowly varying both at the origin and at infinity. Let $J_{0}(n)$ and $J_{\infty}(n)$ denote the set of matrix functions for which condition (2) or (3), respectively, holds for all $\alpha, \beta \in \mathbb{N}_{0}$. Finally, set

$$
J(n)=J_{0}(n) \cap J_{\infty}(n) \cap \mathcal{E}^{-1}(n) .
$$

Let $a(r, \lambda) \in \mathcal{E}^{m}(n)$. The operator

$$
(O p(a) u)(r)=a\left(r, \mathcal{D}_{r}\right) u=\int_{\mathbb{R}} d^{\prime} \lambda \int_{\mathbb{R}_{+}} a(r, \lambda)\left(r \rho^{-1}\right)^{i \lambda} u(\rho) \rho^{-1} d \rho
$$

where $d^{\prime} \lambda=\frac{1}{2 \pi} d \lambda$ and $u \in C_{0}^{\infty}\left(\mathbb{R}_{+}, \mathbb{C}^{n}\right)$, is called the Mellin pseudodifferential operator with the symbol $a(r, \lambda)$. The class of all such operators is denoted by $\operatorname{OPE}^{m}(n)$. The notations $O P \widetilde{\mathcal{E}}(n), O P J_{0}(n), O P J_{\infty}(n), O P J(n)$ have the obvious meaning.

Let $a(r, \rho, \lambda)^{\prime}\left(\in C^{\infty}\left(\mathbb{R}_{+} \times \mathbb{R}_{+} \times \mathbb{R}\right)\right)$ be a matrix function such that

$$
\sup _{\mathbb{R}_{+}^{2} \times \mathbb{R}}\left|\left(r \partial_{r}\right)^{\beta}\left(\rho \partial_{\rho}\right)^{\gamma} \partial_{\lambda}^{\alpha} a_{i j}(r, \rho, \lambda)\right|\langle\lambda\rangle^{\alpha}<\infty
$$

for all $\alpha, \beta, \gamma \in \mathbb{N}_{0}$ and $i, j=1, \ldots, n$. We denote by $\mathcal{E}_{d}^{0}(n)$ the class of all matrix functions satisfying (5).

An operator which is defined by (4) with $a(r, \lambda)$ replaced by $a(r, \rho, \lambda)$ is called a Mellin pseudodifferential operator with a double symbol. Let $O P \mathcal{E}_{d}^{0}(n)$ stand for the class of such operators. We say that a double symbol $a(r, \rho, \lambda)$ is slowly varying if

$$
\left.\begin{array}{l}
\lim _{r \rightarrow+0} \sup _{\rho \in K, \lambda \in \mathbb{R}}\left|\left(r \partial_{r}\right)^{\beta}\left(\rho \partial_{\rho}\right)^{\gamma} \partial_{\lambda}^{\alpha} a_{i j}(r, r \rho, \lambda)\right|\langle\lambda\rangle^{\alpha}=0 \\
\lim _{r \rightarrow+\infty} \sup _{\rho \in K, \lambda \in \mathbb{R}}\left|\left(r \partial_{r}\right)^{\beta}\left(\rho \partial_{\rho}\right)^{\gamma} \partial_{\lambda}^{\alpha} a_{i j}(r, r \rho, \lambda)\right|\langle\lambda\rangle^{\alpha}=0
\end{array}\right\}
$$


for all $\alpha, \beta, \gamma \in \mathbb{N}_{0}$ such that $\beta+\gamma \neq 0$ and for every compact $K \subset \mathbb{R}_{+}$. We denote by $\widetilde{\mathcal{E}}_{d}(n)$ the class of slowly varying double symbols and by $O P \widetilde{\mathcal{E}}_{d}(n)$ the corresponding class of operators.

Throughout what follows we suppose that $1<p<\infty$. Let $L_{p}\left(\mathbb{R}_{+}, d \mu\right)$ be the Lebesgue space on $\mathbb{R}_{+}=(0, \infty)$ with the measure $d \mu(\varrho)=\frac{d \rho}{\ell}$. We denote by $L_{p}^{n}\left(\mathbb{R}_{+}, d \mu\right)$ the Banach space of all measurable complex vector functions $u(r)=\left(u_{1}(r), \ldots, u_{n}(r)\right)$ $\left(r \in \mathbb{R}_{+}\right)$with the norm

$$
\|u\|_{L_{p}^{n}\left(\mathbb{R}_{+}, d \mu\right)}=\left(\sum_{j=1}^{n} \int_{0}^{\infty}\left|u_{j}(r)\right|^{p} d \mu\right)^{\frac{1}{p}}
$$

We also use the following notations: if $X$ is a Banach space, then $\mathcal{L}(X)$ is the Banach algebra of all bounded linear operators on $X$, while $\mathcal{K}(X)$ is the ideal of all compact operators in $\mathcal{L}(X)$.

We now summarize some properties of Mellin pseudodifferential operators we will need in what follows.

Proposition 1.

(a) Every operator $A \in O P \mathcal{E}^{0}(n)$ is bounded in $L_{p}^{n}\left(\mathbb{R}_{+}, d \mu\right)(p \in(1, \infty))$ and we have $\|A\|_{\left.\mathcal{L} L_{p}(\Gamma)\right)} \leq C_{p, s} M$ where

$$
M=\max _{1 \leq i, j \leq n} \sum_{\substack{0 \leq \alpha \leq 1 \\ 0 \leq \beta \leq k}} \sup _{\substack{\mathbb{R}_{+} \times \mathbb{R} \\ 0 \leq \beta}}\left|\left(r \partial_{r}\right)^{\beta} \partial_{\lambda}^{\alpha} a_{i j}(r, \lambda)\right|\langle\lambda\rangle^{\alpha}
$$

and $k, l$ are independent of $A$.

(b) If $A \in O P \mathcal{E}^{0}(n)$ is invertible in $L_{p}^{n}\left(\mathbb{R}_{+}, d \mu\right)(p \in(1, \infty))$, then $A^{-1}$ also belongs to $O P \mathcal{E}^{0}(n)$.

(c) Let $A, B \in O P \widetilde{\mathcal{E}}(n)$. Then $A B \in O P \widetilde{\mathcal{E}}(n)$ and the symbol $\sigma_{A B}(r, \lambda)$ of $A B$ is given by the formula $\sigma_{A B}(r, \lambda)=a(r, \lambda) b(r, \lambda)+t_{1}(r, \lambda)$ where $t_{1}(r, \lambda) \in J(n)$.

(d) Let $A(\in O P \widetilde{\mathcal{E}}(n))$ act on $L_{p}^{n}\left(\mathbb{R}_{+}, d \mu\right) \quad(p \in(1, \infty))$. Then $A^{*}$ belongs to $O P \mathcal{E}(n)$ and $\sigma_{A^{*}}(r, \lambda)$ is given by the formula $\sigma_{A^{*}}(r, \lambda)=a^{*}(r, \lambda)+t_{2}(r, \lambda)$ where $t_{2}(r, \lambda) \in J(n)$.

(e) Let $A$ be a Mellin pseudodifferential operator with a double symbol $a(r, \rho, \lambda) \in$ $\widetilde{S}_{d}(n)$. Then $A \in O P \widetilde{\mathcal{E}}(n)$ and $\sigma_{A}(r, \lambda)=a(r, r, \lambda)+t_{3}(r, \lambda)$ where $t_{3}(r, \lambda) \in J(n)$.

(f) $O P J(n) \subset \mathcal{K}\left(L_{p}^{n}\left(\mathbb{R}_{+}, d \mu\right)\right)$ for all $p \in(1, \infty)$.

2.2 Mellin pseudodifferential operators with analytic symbols in weighted $\boldsymbol{L}_{\boldsymbol{p}}$-spaces. Let $w \in C^{\infty}\left(\mathbb{R}_{+}\right)$. We say that a vector function $u$ belongs to $L_{p}^{n}\left(\mathbb{R}_{+}, w d \mu\right)$ if $\|u\|_{L_{p}^{n}\left(\mathbb{R}_{+}, w d \mu\right)}=\|w u\|_{L_{p}^{n}\left(\mathbb{R}_{+}, d \mu\right)}<\infty$. In what follows we consider weights $w(r)=$ $\exp v(r)$ where the function $v$ satisfies the estimates

$$
\left|\left(r \frac{d}{d r}\right)^{k} v(r)\right|<\infty \quad(k \in \mathbb{N})
$$


Moreover, we require that there is an interval $(c, d) \ni 0$ such that the function $\kappa_{w}(r)=$ $r v^{\prime}(r)$ satisfies the condition

$$
c<\inf _{r \in \mathbb{R}_{+}} \kappa_{w}(r) \leq \sup _{r \in \mathbb{R}_{+}} \kappa_{w}(r)<d .
$$

We say that a weight $w$ is slowly varying on $\mathbb{R}_{+}$if conditions $(6)-(7)$ hold, $\lim _{r \rightarrow 0} r \kappa_{w}^{\prime}(r)$ $=0$ and $\lim _{r \rightarrow \infty} r \kappa_{w}^{\prime}(r)=0$. We denote by $\mathcal{R}(c, d)$ the class of slowly varying weights.

Definition 2. We say that a matrix-function $a(r, \rho, \lambda)$ is in $\mathcal{E}_{d}^{0}(n,(c, d))$ if $a(r, \rho, \lambda)$ is analytically continued with respect to $\lambda$ into the strip $\Pi=\{\lambda \in \mathbb{C}: \operatorname{Im} \lambda \in(c, d)\}$ and

$$
\sup _{\mathbb{R}_{+}^{2} \times \Pi}\left|\left(r \partial_{r}\right)^{\beta}\left(\rho \partial_{\rho}\right)^{\gamma} \partial_{\lambda}^{\alpha} a_{i j}(r, \rho, \lambda)\right|\langle\lambda)^{\alpha}<\infty .
$$

We let $O P \mathcal{E}_{d}^{0}(n,(c, d))$ denote the corresponding class of Mellin pseudodifferential operators with analytic symbols.

The class $O P \widetilde{\mathcal{E}}_{d}(n,(c, d))$ of Mellin pseudodifferential operators with slowly varying double analytic symbols is introduced in evident way.

\section{Proposition 3.}

(a) Let $a(r, \rho, \lambda) \in \mathcal{E}_{d}^{0}(n,(c, d))$ and let the weight $w$ satisfy conditions $(6)-(7)$. Then $w O p(a) w^{-1} \in \mathcal{E}_{d}^{0}(n)$ and $w O p(a) w^{-1}=O p\left(a\left(r, \rho, \lambda+i \vartheta_{w}(r, \rho)\right)\right.$ where $\vartheta_{w}(r, \rho)=$ $\int_{0}^{1} \kappa_{w}\left(r^{1-r} \rho^{r}\right) d \tau$ (it is easy to see that condition $(7)$ yields $\vartheta_{w}(r, \rho) \in(c, d)$ for all $\left.r, \rho \in \mathbb{R}_{+}\right)$.

(b) Let $O p(a) \in O P \widetilde{\mathcal{E}}_{d}(n,(c, d))$ and $w \in \mathcal{R}(c, d)$. Then $w O p(a) w^{-1} \in O P \tilde{\mathcal{E}}_{d}(n)$ and $w O p(a) w^{-1}=O p\left(a\left(r, r, \lambda+i \kappa_{w}(r)\right)\right)+q(r, \lambda)$ where $q(r, \lambda) \in J(n)$.

Corollary 4. Let $O p(a) \in O P \mathcal{E}_{d}^{0}(n,(c, d))$ and suppose a weight $w$ satisfies conditions (6) - (7). Then $A$ is bounded in $L_{p}^{n}\left(\mathbb{R}_{+}, w d \mu\right)$.

\subsection{Local invertibility. First we give the following definition.}

Definition 5. Let $A \in \mathcal{L}\left(L_{p}^{n}\left(\mathbb{R}_{+}, w d \mu\right)\right)$. We say that $A$ is a locally invertible operator at the point 0 if there exist $R>0$ and operators $B^{\prime}, B^{\prime \prime} \in \mathcal{L}\left(L_{p}^{n}\left(\mathbb{R}_{+}, w d \mu\right)\right)$ such that $B^{\prime} A_{\chi_{R}}=\chi_{R}$ and $\chi_{R} A B^{\prime \prime}=\chi_{R}$ where $\chi_{R}$ is the operator of multiplication by the characteristic function of the segment $[0, R]$. In the same way we define local invertibility at the point $+\infty$.

Theorem 6. Let $A=a\left(r, \mathcal{D}_{r}\right) \in O P \widetilde{\mathcal{E}}(n,(c, d))$ and $w \in \mathcal{R}(c, d)$. Then the operator $A: L_{p}^{n}\left(\mathbb{R}_{+}, w d \mu\right) \rightarrow L_{p}^{n}\left(\mathbb{R}_{+}, w d \mu\right)$ is locally invertible at the origin or at infinity if and only if

$$
\lim _{\varepsilon \rightarrow 0} \inf _{(0, e) \times \mathbb{R}}\left|\operatorname{det} a\left(r, \lambda+i \kappa_{w}(r)\right)\right|>0
$$

or

$$
\lim _{R \rightarrow+\infty} \inf _{R, \infty) \times \mathbb{R}}\left|\operatorname{det} a\left(r, \lambda+i \kappa_{w}(r)\right)\right|>0
$$

respectively.

2.4 Fredholmness and index. Here we formulate the following result. 


\section{Theorem 7.}

(a) Let $A=O p(a) \in O P \widetilde{\mathcal{E}}(n,(c, d))$ and $w \in \mathcal{R}(c, d)$. Then the operator $A$ : $L_{p}^{n}\left(\mathbb{R}_{+}, w d \mu\right) \rightarrow L_{p}^{n}\left(\mathbb{R}_{+}, w d \mu\right)$ is Fredholm if and only if conditions $(8)-(9)$ are fulfilled and

$$
\lim _{R \rightarrow+\infty} \inf _{\mathbb{R} \times(R, \infty)}|\operatorname{det} a(r, \lambda)|>0
$$

(b) If $A \in O P \tilde{\mathcal{E}}(n,(c, d))$ is a Fredholm operator, then

$$
\operatorname{Ind} A=-\frac{1}{2 \pi}\left[\arg a\left(r, \lambda+i \kappa_{w}(r)\right)\right]_{\Gamma\left(R^{\prime}, R^{\prime \prime}\right)}
$$

where $\Gamma\left(R^{\prime}, R^{\prime \prime}\right)$ is the positively oriented boundary of the rectangle

$$
\Pi\left(R^{\prime}, R^{\prime \prime}\right)=\left\{(r, \lambda) \in \mathbb{R}_{+} \times \mathbb{R}: \frac{1}{R^{\prime}}<r<R^{\prime} \text { and }|\lambda|<R^{\prime \prime}\right\} .
$$

Here the numbers $R^{\prime}, R^{\prime \prime}$ are such that $a^{-1}\left(r, \lambda+i \kappa_{w}(r)\right)$ exists for all points of the domain $\left(\mathbb{R}_{+} \times \mathbb{R}\right) \backslash \Pi\left(R^{\prime}, R^{\prime \prime}\right)$.

\section{Potential operators on simple curves}

We now consider the potential type operator

$$
A u(x)=\frac{1}{\pi} \int_{\gamma} \frac{(\nu(y), x-y)}{|x-y|^{2}} g(x, y) u(y) d l_{y} \quad(x \in \gamma)
$$

on a simple unbounded curve $\gamma$. We suppose that $\gamma$ has the parametrization

$$
\gamma=\{x \in \mathbb{C}: x=t \exp i \theta(t)\} \quad\left(t \in \mathbb{R}_{+}\right)
$$

where $\theta$ is a real-valued $C^{\infty}$-function on $\mathbb{R}_{+}$satisfying the conditions

$$
\left.\begin{array}{l}
\sup _{\mathbb{R}_{+}}\left|\left(t \frac{d}{d t}\right)^{k} \theta(t)\right|<\infty \quad(k \in \mathbb{N}) \\
\lim _{t \rightarrow+\infty}\left(t \frac{d}{d t}\right)^{2} \theta(t)=0 \\
\lim _{t \rightarrow+\infty}\left(t \frac{d}{d t}\right)^{2} \theta(t)=0 .
\end{array}\right\}
$$

The simplest example of such a curve is the logarithmic spiral $x=t \exp (i \delta \ln t) \quad(t \in$ $\mathbb{R}_{+}$) with the twisting coefficient $\delta \in \mathbb{R}$. More complicated examples are provided by curves with a variable twisting coefficient $\delta(t): x=t \exp (i \delta(t) \ln t)\left(t \in \mathbb{R}_{+}\right)$where $\delta(t)=\sin \left(\ln ^{\alpha}\langle\ln \rangle\right)$ with $\alpha \in(0,1)$. It is easy to check that $\theta(t)=\delta(t) \ln t$ satisfies the above conditions (13).

We suppose that $g(x, y)=\left(g_{k l}(x, y)\right)_{k, l=1}^{n}$ where $g_{k l} \in C^{\infty}(\gamma \times \gamma)$ and the functions

$$
\tilde{g}_{k, l}(t, \tau)=g_{k, l}(t \exp i \theta(t), \tau \exp i \theta(\tau))
$$


are subject to the coditions

$$
\sup _{\mathbb{R}_{+} \times \mathbb{R}_{+}}\left|\left(t \partial_{t}\right)^{\alpha}\left(\tau \partial_{\tau}\right)^{\beta} \tilde{g}_{k, l}(t, \tau)\right|<\infty \quad\left(\alpha, \beta \in \mathbb{N}_{0}\right) .
$$

Moreover, we require that

$$
\begin{aligned}
& \limsup _{t \rightarrow 0} \sup _{\tau}\left|t \frac{\partial}{\partial t} \tilde{g}_{k, l}(t, \tau)\right|=\lim _{t \rightarrow \infty} \sup _{\tau}\left|t \frac{\partial}{\partial t} \tilde{g}_{k, l}(t, \tau)\right|=0 \\
& \lim _{l \rightarrow 0} \sup _{t}\left|\tau \frac{\partial}{\partial \tau} \tilde{g}_{k, l}(t, \tau)\right|=\lim _{t \rightarrow \infty} \sup _{t}\left|\tau \frac{\partial}{\partial \tau} \tilde{g}_{k, l}(t, \tau)\right|=0 .
\end{aligned}
$$

Let $\Phi: C_{0}^{\infty}\left(\gamma, \mathbb{C}^{n}\right) \rightarrow C_{0}^{\infty}\left(\mathbb{R}_{+}, \mathbb{C}^{n}\right)$ be the mapping acting by the formula

$$
\tilde{u}(t)=(\Phi u)(t)=t^{\frac{1}{p}} u(t \exp i \theta(t)) \quad\left(t \in \mathbb{R}_{+}\right) .
$$

Clearly, $\Phi$ can be continued to an isometric isomorphism of $L_{p}\left(\gamma ; \mathbb{C}^{n}\right)$ on $L_{p}\left(\mathbb{R}_{+}, \mathbb{C}^{n}, d \mu\right)$.

Proposition 8. Let $A$ be an operator of the form (11). Then $A_{\Phi}=\Phi A \Phi^{-1}$ is a Mellin pseudodifferential operator in the class $O P \widetilde{\mathcal{E}}(n)$ with the symbol

$$
\sigma_{A_{+}}(t, \lambda)=\frac{i}{2} \frac{\sin \frac{2 \pi \delta_{\theta}(t)\left(\lambda+i \frac{1}{p}\right)}{1+\delta_{p}^{2}(t)}}{\sinh \frac{\pi\left(\lambda+i \frac{1}{p}\right)}{1+i \delta_{\theta}(t)} \sinh \frac{\pi\left(\lambda+i \frac{1}{p}\right)}{1-i \delta_{\theta}(t)}} \tilde{g}(t, t)+q(t, \lambda)
$$

where $\delta_{\theta}(t)=t \theta^{\prime}(t), q(t, \lambda) \in J_{0}$. form

Proof. After the change of variables $y=\tau \exp i \theta(\tau)$, the vector $\nu(y)$ assumes the

$$
\nu(z+\tau \exp i \theta(\tau))=\frac{\left(1+i \tau \theta^{\prime}(\tau)\right) \exp i\left(\theta(\tau)-\frac{\pi}{2}\right)}{\left(1+\left(\tau \theta^{\prime}(\tau)\right)^{2}\right)^{\frac{1}{2}}}=d(\tau) \exp i \theta(\tau)
$$

where

$$
d(\tau)=-i \frac{1+i \tau \theta^{\prime}(\tau)}{\left(1+\left(\tau \theta^{\prime}(\tau)\right)^{2}\right)^{\frac{1}{2}}}
$$

Further,

$$
\begin{aligned}
\frac{(\nu(y), x-y)}{|x-y|^{2}} & =\frac{\operatorname{Re}[\nu(y) \overline{(x-y)}]}{|x-y|^{2}} \\
& =\frac{1}{2} \frac{\nu(y) \overline{(x-y)}+\overline{\nu(y)}(x-y)}{|x-y|^{2}} \\
& =\frac{1}{2}\left(\frac{\nu(y)}{x-y}+\frac{\overline{\nu(y)}}{\overline{x-y}}\right) .
\end{aligned}
$$

It follows from (20) that

$$
\frac{(\nu(y), x-y)}{|x-y|^{2}}=\frac{1}{2}\left(\frac{d(\tau)}{t \exp i(\theta(t)-\theta(\tau))-\tau}+\frac{\overline{d(\tau)}}{t \exp -i(\theta(t)-\theta(\tau))-\tau}\right) .
$$


Taking into account (13) we obtain that $d(\tau)$ satisfies the estimates $\sup _{\mathbb{R}_{+}}\left|\left(t \frac{d}{d t}\right)^{k} d(t)\right|<$ $\infty \quad\left(k \in \mathbb{N}_{0}\right)$ and that $\lim _{t \rightarrow+0}\left(t \frac{d}{d t}\right) d(t)=0$ as well as $\lim _{t \rightarrow \infty}\left(t \frac{d}{d t}\right) d(t)=0$. Let us introduce the function

$$
m(\theta)(t, \tau)=\frac{\theta(t)-\theta(\tau)}{\ln t-\ln \tau}=\int_{0}^{1} t^{1-r} \tau^{r} \theta^{\prime}\left(t^{1-r} \tau^{r}\right) d r .
$$

It is easy to check that $m(\theta)(t, \tau)$ satisfies conditions (14) - (16). Formula (22) yields

$$
\exp i(\theta(t)-\theta(\tau))=\left(t \tau^{-1}\right)^{i m(\theta)(t, \tau)} \text {. }
$$

Applying formulas (21) and (23) we see that $A_{\Phi}$ is the integral operator

$$
\left(A_{\Phi} v\right)(t)=\frac{1}{2} \int_{\mathbb{R}_{+}}(k(t, \tau)+\overline{k(t, \tau)}) \tilde{g}(t, \tau) v(\tau) \frac{d \tau}{\tau}
$$

where $k(t, \tau)=i \frac{1+i r \theta^{\prime}(\tau)}{\left(1-\left(t \tau^{-1}\right)^{1+i m(D)(1, \tau)}\right)}$. If $\operatorname{Re} \xi \geq 1$, then

$$
\frac{1}{\pi i} \frac{\xi}{1-x^{\xi}}=\frac{1}{2 \pi} \int_{\mathbb{R}} \operatorname{coth} \pi\left(\frac{\lambda}{\xi}\right) x^{i \lambda} d \lambda \quad\left(x \in \mathbb{R}_{+}\right)
$$

(see [15: p. 414]). Using (24), (18) - (19) and the equality $d l=\left(1+\left(\tau \theta^{\prime}(\tau)\right)^{2}\right)^{\frac{1}{2}} d \tau$ we arrive at the representation

$$
\left(A_{\Phi} v\right)(t)=\frac{1}{2 \pi} \int_{\mathbb{R}} d \lambda \int_{\mathbb{R}_{+}} a\left(t, \tau, \lambda+i \frac{1}{p}\right)\left(t \tau^{-1}\right)^{i \lambda} v(\tau) \frac{d \tau}{\tau}
$$

where

$$
a(t, \tau, \lambda)=\frac{1}{2}\left(a_{1}(t, \tau, \lambda)+\overline{a_{1}(t, \tau, \lambda)}\right) \tilde{g}(t, \tau)
$$

and

$$
a_{1}(t, \tau, \lambda)=-\frac{1+i \tau \theta^{\prime}(\tau)}{1+\operatorname{im}(\theta)(t, \tau)} \operatorname{coth} \pi\left(\frac{\lambda}{1+\operatorname{im}(\theta)(t, \tau)}\right) .
$$

From estimates (13) and (15) - (16) we infer that $a(t, \tau, \lambda) \in \mathcal{E}_{d}^{0}(n)$. Hence, by Proposition $1 /(\mathrm{e}), A_{\Phi}$ is a Mellin pseudodifferential operator with the symbol

$$
a(t, t, \lambda)=\frac{1}{2}\left[\operatorname{coth} \pi\left(\frac{\lambda+i \frac{1}{p}}{1+i \tau \theta^{\prime}(\tau)}\right)-\operatorname{coth} \pi\left(\frac{\lambda+i \frac{1}{p}}{1-i \tau \theta^{\prime}(\tau)}\right)\right] \tilde{g}(t, \tau)+q(t, \lambda)
$$

where $q(t, \lambda) \in J_{0}$. Finally, the identity $\operatorname{coth} a-\operatorname{coth} b=\frac{\sinh (b-a)}{\sinh a \sinh b}$ in conjunction with (25) gives formula (17)

Let us now consider $A$ as an operator acting in the space $L_{p}^{n}(\gamma, w)$ with the norm $\|u\|_{L_{p}^{n}(\gamma, w)}=\|w u\|_{L_{p}^{n}(\gamma)}$. We suppose that the weight $w$ satisfies the condition

$$
\widetilde{w}(t)=w(t \exp i \theta(t)) \in \mathcal{R}\left(-\frac{1}{p}, 1-\frac{1}{p}\right) .
$$

This condition is similar the well-known Hunt-Muckenhoupt condition (see, for instance, [1]). 
Corollary 9. Let $A$ be an operator of the form (11) and let $w$ be a weight on $\gamma$ satisfying condition (26). Then $A_{\Phi, w}=\Phi w A w^{-1} \Phi^{-1}$ is a Mellin pseudodifferential operator in the class $O P \widetilde{\mathcal{E}}$. Its symbol is given by the formula

$$
\sigma_{A+, w}(t, \lambda)=\frac{i}{2} \frac{\sin \frac{2 \pi \delta_{\theta}(t)\left(\lambda+i\left(\frac{1}{p}+\kappa_{\bar{u}}(t)\right)\right)}{1+\delta_{\dot{q}}^{2}(t)}}{\sinh \frac{\pi\left(\lambda+i\left(\frac{1}{p}+\kappa_{\dot{w}}(t)\right)\right)}{1+i \delta_{\theta}(t)} \sinh \frac{\pi\left(\lambda+i\left(\frac{1}{p}+\kappa_{\dot{w}}(t)\right)\right)}{1-i \delta_{\theta}(t)}} \tilde{g}(t, \tau)+q(t, \lambda)
$$

where $q(t, \lambda) \in J_{0}$ and $\kappa_{\bar{w}}(t)=t \frac{\bar{w}^{\prime}(t)}{\bar{w}(t)}$.

Proof. This corollary follows from Proposition 3

Corollary 10. Let $A$ be an operator of type (11) and let $w$ be a weight on $\gamma$ satis. fying condition (26). Then $A: L_{p}^{n}(\gamma, w) \rightarrow L_{p}^{n}(\gamma, w)$ is a compact operator if and only if

$$
\lim _{t \rightarrow 0} \sigma_{A_{\uparrow, w}}(t, \lambda)=\lim _{t \rightarrow \infty} \sigma_{A_{\downarrow, \omega}}(t, \lambda)=0
$$

uniformly with respect to $\lambda \in \mathbb{R}$.

Corollary 10 tells us that there are two reasons for $A: L_{p}^{n}(\gamma, w) \rightarrow L_{p}^{n}(\gamma, w)$ to be a compact operator:

1) $\lim _{t \rightarrow 0} \tilde{g}(t, t)=\lim _{t \rightarrow \infty} \tilde{g}(t, t)=0$

2) $\lim _{t \rightarrow 0} \delta_{\theta}(t)=\lim _{t \rightarrow \infty} \delta_{\theta}(t)=0$.

Let

$$
A u(x)=\frac{1}{\pi} \int_{\gamma} \frac{(\nu(y), x-y)}{|x-y|^{2}} u(y) d l_{y} \quad(x \in \gamma)
$$

be the potential operator of the double layer. Then $A$ is compact if and only if condition 2) holds. Let $\gamma$ be a bounded curve with the parametrization $\gamma=\{x \in \mathbb{C}: x=$ $\left.t \exp i \theta(t)), t \in\left[0, t_{0}\right]\right\}$ where $\theta$ satisfies condition $(13)_{1}$ on $\left[0, t_{0}\right]$. Suppose also that condition (13) $)_{2}$ is fulfilled. Then $A: L_{p}^{n}(\gamma, w) \rightarrow L_{p}^{n}(\gamma, w)$ is a compact operator if and only if $\lim _{t \rightarrow 0} \delta_{\theta}(t)$.

Let us now consider the operator

$$
B u(x)=a(x) u(x)+\frac{1}{\pi} \int_{\gamma} \frac{(\nu(y), x-y)}{|x-y|^{2}} g(x, y) u(y) d l_{y} \quad(x \in \gamma)
$$

where $a(x)=\left(a_{k, l}(x)\right)_{k, l=1}^{n}$ and $\tilde{a}_{k, l}(t)=a_{k, l}(t \exp i \theta(t))$ satisfy the same estimates with respect to $t$ as $\tilde{g}_{k, l}(t, \tau)$. It follows from Corollary 9 that, up to a symbol $q(t, \lambda) \in J_{0}$, $B_{\Phi, w}$ is a Mellin pseudodifferential operator with the symbol

$$
\sigma_{B_{\star, w}}(t, \lambda)=\tilde{a}(t)+\sigma_{A \downarrow, w}(t, \lambda)
$$

where $\sigma_{A \uparrow, \omega}(t, \lambda)$ is given by formula (27).

The next two theorems follow from Theorem 7 and Proposition 3. 
Theorem 11. $B: L_{p}^{n}(\gamma, w) \rightarrow L_{p}^{n}(\gamma, w)$ is a Fredholm operator if and only if

(i) $\inf _{x \in \gamma}|\operatorname{det} a(x)|>0$

(ii) $\underline{\lim }_{t \rightarrow 0} \inf _{\lambda \in \mathbb{R}}\left|\operatorname{det} \sigma_{B_{\uparrow, w}}(t, \lambda)\right|>0$ and $\underline{\lim }_{t \rightarrow \infty} \inf _{\lambda \in \mathbb{R}}\left|\operatorname{det} \sigma_{B_{\uparrow, w}}(t, \lambda)\right|>0$.

If conditions ( $i)$ and (ii) are fulfilled, then

$$
\text { Ind } B=-\frac{1}{2 \pi}\left[\operatorname{argdet} \sigma_{B_{4, \omega}}(t, \lambda)\right]_{\Gamma\left(R^{\prime}, R^{\prime \prime}\right)}
$$

where $\Gamma\left(R^{\prime}, R^{\prime \prime}\right)$ is the positively oriented boundary of the rectangle

$$
\Pi\left(R^{\prime}, R^{\prime \prime}\right)=\left\{(r, \lambda) \in \mathbb{R}_{+} \times \mathbb{R}: \frac{1}{R^{\prime}}<r<R^{\prime} \text { and }|\lambda|<R^{\prime \prime}\right\}
$$

and where the numbers $R^{\prime}, R^{\prime \prime}$ are chosen so that $\left[\sigma_{A \uparrow, w}(t, \lambda)\right]^{-1}$ exists for all points in the domain $\left(\mathbb{R}_{+} \times \mathbb{R}\right) \backslash \Pi\left(R^{\prime}, R^{\prime \prime}\right)$.

Theorem 12. The operator $B: L_{p}^{n}(\gamma, w) \rightarrow L_{p}^{n}(\gamma, w)$ is locally invertible at the point 0 if and only if $\underline{\lim }_{t \rightarrow 0} \inf \lambda_{\lambda \in \mathbb{R}}\left|\operatorname{det} \sigma_{B_{\bullet}, w}(t, \lambda)\right|>0$.

The local spectrum of an operator $A: L_{p}^{n}(\gamma, w) \rightarrow L_{p}^{n}(\gamma, w)$ at a point $x \in \gamma$ is defined as the set of all points $\varsigma \in \mathbb{C}$ for which $A-\varsigma I$ is not locally invertible at the point $x$. We denote the local spectrum of $A$ at the point $x$ by $S p_{x} A$.

Theorem 13. A point $\varsigma$ belongs to $S p_{2} A$ if and only if there exists a sequence $t_{m} \rightarrow 0$ such that $\lim _{m \rightarrow \infty} \inf _{\lambda \in \mathbb{R}}\left|\operatorname{det}\left(\sigma_{A_{\bullet}, w}\left(t_{m}, \lambda\right)-\varsigma I\right)\right|=0$. Thus, the local spectrum of $A$ at the singular point 0 is

$$
S p_{0} A_{\Gamma}=\bigcup\left\{\varsigma \in \mathbb{C}: \operatorname{det}\left(\tilde{\sigma}_{A_{\uparrow, \omega}}(\lambda)-\varsigma I\right)=0 \text { for some } \lambda \in \mathbb{R} \cup\{\infty\}\right\}
$$

where $\operatorname{det}\left(\tilde{\sigma}_{A_{\uparrow, w}}(\lambda)-\varsigma I\right)$ denotes the set of all partial limits of $\operatorname{det}\left(\sigma_{A_{\uparrow, w}}(t, \lambda)-\varsigma I\right)$ as $t \rightarrow 0$.

From the previous theorem we learn in particular that the local spectrum of $A$ at the point 0 can be a set of positive planar Lebsegue measure and that its structure is defined by the behavior of the curve, of the coefficients, and the weight at the point zero.

\section{Potential type operators on Jordan curves with vorticity points}

In this section we consider potential operators on oriented closed Jordan curves $\Gamma$ in the complex plane $\mathbb{C}$. We will suppose that $\Gamma$ has a finite set $F$ of singular points and that $\Gamma \backslash F$ is locally a Lyapunov curve. that

If $z \in F$ is a singular point, then there is a neighborhood $U^{z}$ of the point $z$ such

$$
\Gamma \cap U_{ \pm}^{z}=\left\{x=z \pm t \exp i \omega_{ \pm}^{z}(t): t \in[0, s]\right\}
$$


where $U_{+}^{z}$ and $U_{-}^{z}$ is a right side and left side half-neighborhood, respectively, of the point $z$. We suppose that

$$
\omega_{ \pm}^{z}(t)=\theta_{0}^{z}(t)+\theta_{ \pm}^{z}(t) \quad(t \in(0, s])
$$

where the functions $\theta_{0}^{z}$ and $\theta_{ \pm}^{z}$ satisfy estimate $(13)_{1}$ on the interval $[0, s]$ and equality $(13)_{2}$. Moreover, we suppose that

$$
0 \leq \theta_{-}^{z}(t) \leq M_{-}<m_{+} \leq \theta_{+}^{z}(t) \leq M_{+}<2 \pi \quad(z \in F) .
$$

From (28) it follows that

$$
\lim _{t \rightarrow 0} t \frac{d \theta_{ \pm}^{z}(t)}{d t}=0
$$

Hence, the function $\theta_{0}^{z}$ describes the rotation of the curve $\Gamma$ at the point $z$, while the functions $\theta_{ \pm}^{z}$ characterize the oscillation of the curve at that point. In the case where

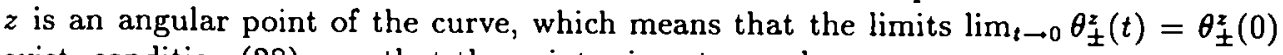
exist, condition (28) says that the point $z$ is not a peak.

Let

$$
A_{\Gamma} u(x)=\frac{1}{\pi} \int_{\Gamma} \frac{(\nu(y), x-y)}{|x-y|^{2}} g(x, y) u(y) d l_{y} \quad(x \in \Gamma)
$$

be a potential operator on the curve $\Gamma$, assume the matrix function $g(x, y)$ is bounded on $\Gamma$ and continuous on $\Gamma \backslash F$, and also assume that the matrix functions

$$
\begin{aligned}
& g_{++}^{z}(t, \tau)=g\left(z+t \exp i \omega_{+}^{z}(t), z+\tau \exp i \omega_{+}^{z}(\tau)\right) \\
& g_{+-}^{z}(t, \tau)=g\left(z+t \exp i \omega_{+}^{z}(t), z-\tau \exp i \omega_{-}^{z}(\tau)\right) \\
& g_{-+}^{z}(t, \tau)=g\left(z-t \exp i \omega_{-}^{z}(t), z+\tau \exp i \omega_{+}^{z}(\tau)\right) \\
& g_{--}^{z}(t, \tau)=g\left(z-t \exp i \omega_{-}^{z}(t), z-\tau \exp i \omega_{-}^{z}(\tau)\right)
\end{aligned}
$$

have entries satisfying conditions (14) - (15) in a neighborhood of the points $t=0$ and $\tau=0$.

We consider the operator $A_{\Gamma}$ in the weighted space $L_{p}^{n}(\Gamma, w)$ where $1<p<\infty$ and $w$ is a positive continuous function on $\Gamma \backslash F$. In a neighborhood of a singular point $z$ the weight $w$ is supposed to admit a representation $w(x)=\exp v^{2}(|x-z|) \quad(|x-z|<s)$ where the function $v^{z}$ satisfies estimates $(6)$ on $(0, s)$ and $\lim _{t \rightarrow 0}\left(t \frac{d}{d t}\right)^{2} v^{z}(t)=0$. As above (see condition (26)) we suppose that $\tilde{w}(t)=\exp v^{z}(t) \in \mathcal{R}\left(-\frac{1}{p}, 1-\frac{1}{p}\right)$. Put $\beta^{z}(t)=t \frac{d v^{z}(t)}{d t}, \delta^{z}(t)=t \frac{d \theta_{0}^{2}(t)}{d t}$ and $\theta^{z}(t)=\theta_{+}^{z}(t)-\theta_{-}^{z}(t)$.

Example 14. Let $\omega_{+}^{z}(t)=\delta^{z}(t) \ln t+\theta_{+}^{z}+\frac{\varepsilon}{2} \sin \ln \langle\ln t\rangle$ and $\omega_{-}^{z}(t)=\delta^{z}(t) \ln t+\theta_{-}^{z}$ where $\delta^{z}(t)=f\left(\ln ^{\alpha}(\ln t\rangle\right)$ with $0<\alpha<1, f \in C_{b}^{\infty}\left(\mathbb{R}_{+}\right)$and $\varepsilon<\theta_{+}^{z}-\theta_{-}^{z}<2 \pi-\varepsilon$. Then all the above conditions for $\Gamma$ are fulfilled.

Let $v^{z}(t)=\beta^{z}(t) \ln t$, where $\beta^{z}$ satisfies the same conditions as $\delta^{z}$ and $-\frac{1}{p}<$ $\inf _{(0, s]} \beta^{z}(t) \leq \sup _{(0, s]} \beta^{z}(t)<1-\frac{1}{p}$. Then $\tilde{w}(t)=\exp v^{z}(t) \in \mathbb{R}\left(-\frac{1}{p}, 1-\frac{1}{p}\right)$. formula

We introduce the isometric isomorphism $\Phi_{z}: L_{p}^{n}\left(\Gamma \cap U_{z}, w\right) \rightarrow L_{p}^{2 n}([0, s], d \mu)$ by the

$$
\Phi_{z} f(t)=\left(t^{\frac{1}{p}} e^{v(t)} f\left(z+t \exp i \omega_{+}^{z}(t)\right), t^{\frac{1}{p}} e^{v(t)} f\left(z-t \exp i \omega_{-}^{z-}(t)\right)\right)
$$

for $t \in(0, s]$. 
Proposition 15. Let $\chi_{z}(x)$ be a cut-off function of the point $z$, that is, $\chi_{z}(x) \in$ $C^{\infty}(\Gamma), \chi_{z}(x)=1$ in a neighborhood of the point $z$, and $\operatorname{supp} \chi_{z}(x) \subset U_{z}$. Then $\Phi_{z} \chi_{2} A \chi_{z} \Phi_{z}^{-1}$ is a Mellin pseudodifferential operator in the class $O P \tilde{S}(2 n)$. Its symbol $\sigma^{2}(A)(t, \lambda)$ is given up to a symbol in the class $J_{0}$ by the formula

$$
\sigma^{z}(A)(t, \lambda)=\left(\begin{array}{ll}
a_{++}^{z}(t, \lambda) & a_{-+}^{z}(t, \lambda) \\
a_{+-}^{z}(t, \lambda) & a_{--}^{z}(t, \lambda)
\end{array}\right)
$$

where

$$
\begin{aligned}
& a_{++}^{z}(t, \lambda)=\frac{i}{2} \frac{\sin \frac{2 \pi \delta^{2}(t)\left(\lambda+i\left(\frac{1}{p}+\beta^{2}(t)\right)\right)}{1+\delta^{z}(t)^{2}}}{\sinh \frac{\pi\left(\lambda+i\left(\frac{1}{p}+\beta^{2}(t)\right)\right)}{1+i \delta^{z}(t)} \sinh \frac{\pi\left(\lambda+i\left(\frac{1}{p}+\beta^{2}(t)\right)\right)}{1-i \delta^{z}(t)}} g_{++}^{z}(t, t) \\
& a_{--}^{z}(t, \lambda)=-\frac{i}{2} \frac{\sin \frac{2 \pi \delta^{2}(t)\left(\lambda+i\left(\frac{1}{p}+\beta^{2}(t)\right)\right)}{1+\delta^{2}(t)^{2}}}{\sinh \frac{\pi\left(\lambda+i\left(\frac{1}{p}+\beta^{2}(t)\right)\right)}{1+i \delta^{2}(t)} \sinh \frac{\pi\left(\lambda+i\left(\frac{1}{p}+\beta^{2}(t)\right)\right)}{1-i \delta^{2}(t)}} g_{--}^{z}(t, t) \\
& a_{-+}^{z}(t, \lambda)=\frac{1}{2}\left[\frac{\exp \left(\theta^{z}(t)-\pi\right) \frac{\lambda+i\left(\frac{1}{p}+\beta^{2}(t)\right)}{1+i \delta^{2}(t)}}{\sinh \frac{\pi\left(\lambda+i\left(\frac{1}{p}+\beta^{2}(t)\right)\right)}{1+i \delta^{2}(t)}}-\frac{\exp -\left(\theta^{z}(t)-\pi\right) \frac{\lambda+i\left(\frac{1}{p}+\beta^{2}(t)\right)}{1-i \delta^{2}(t)}}{\sinh \frac{\pi\left(\lambda+i\left(\frac{1}{p}+\beta^{2}(t)\right)\right)}{1-i \delta^{2}(t)}}\right] g_{-+}^{z}(t, t) \\
& a_{+-}^{z}(t, \lambda)=-\frac{1}{2}\left[\frac{\exp -\left(\theta^{z}(t)-\pi\right) \frac{\lambda+i\left(\frac{1}{p}+\beta^{2}(t)\right)}{1+i \delta^{2}(t)}}{\sinh \frac{\pi\left(\lambda+i\left(\frac{1}{p}+\beta^{2}(t)\right)\right)}{1+i \delta^{2}(t)}}-\frac{\exp \left(\theta^{z}(t)-\pi\right) \frac{\lambda+i\left(\frac{1}{p}+\beta^{z}(t)\right)}{1-i \delta^{z}(t)}}{\sinh \frac{\pi\left(\lambda+i\left(\frac{1}{p}+\beta^{2}(t)\right)\right)}{1-i \delta^{2}(t)}}\right] g_{+-}^{z}(t, t) .
\end{aligned}
$$

Proof. The expressions for $a_{++}^{z}(t, \lambda)$ and for $a_{--}^{z}(t, \lambda)$ follow from Corollary 9 . The formulas for $a_{-+}^{z}(t, \lambda)$ and $a_{+-}^{z}(t, \lambda)$ can be proved as in [14] (see the proof of Theorem 3.18 there). We remark that the proofs are based on the formula (see $[14,15])$

$$
J(\lambda, a, \xi)=\frac{a \xi}{\pi i} \int_{0}^{\infty}\left(a-t^{\xi}\right)^{-1} t^{-i \lambda-1} d t=\frac{2 a \exp \left(-i \frac{\lambda}{\xi}-1\right) \ln a}{\exp \left(2 \pi \frac{\lambda}{\xi}\right)-1} \quad\left(a \in \mathbb{C} \backslash \overline{\mathbb{R}}_{+}\right)
$$

where $\operatorname{Im} \lambda \in(0,1)$ and $\operatorname{Re} \xi \geq 1$

Theorem 16. The operator $A_{\Gamma}: L_{p}^{n}(\Gamma, w) \rightarrow L_{p}^{n}(\Gamma, w)(1<p<\infty)$ is bounded.

Proof. Let $\sum_{j=1}^{N} \chi_{j}(x)=1 \quad(x \in \Gamma)$ be a $C^{\infty}$-partition of unity on $\Gamma$ and let $\psi_{j} \in C^{\infty}(\Gamma)$ satisfy $\psi_{j}(x) \chi_{j}(x)=\chi_{j}(x)$. Then

$$
A_{\Gamma}=\sum_{j=1}^{N} \chi_{j} A_{\Gamma}=\sum_{j=1}^{N} \chi_{j} A_{\Gamma} \psi_{j}+\sum_{j=1}^{N} \chi_{j} A_{\Gamma}\left(1-\psi_{j}\right)
$$

The operator $\sum_{j=1}^{N} \chi_{j} A_{\Gamma}\left(1-\psi_{j}\right)$ is bounded because supp $\chi_{j} \cap \operatorname{supp} \psi_{j}=0$. If $\operatorname{supp} \chi_{j}$ contains a singular point, the boundedness of $\chi_{j} A_{\Gamma} \psi_{j}$ follows from Proposition $1 /(\mathrm{a})$. In the opposite case, $\chi_{j} A_{\Gamma} \psi_{j}$ is bounded as a potential type operator on a Lyapunov curve 
Remark 1. Let the curve $\Gamma$ have zero rotation at a singular point $z \in F$, that is, assume $\lim _{t \rightarrow 0} \delta^{z}(t)=0$. Then the formulas for the symbol $\sigma^{2}\left(A_{\Gamma}\right)(t, \lambda)$ can be simplified. Namely, in this case

$$
\sigma^{z}\left(A_{\Gamma}\right)(t, \lambda)=\left(\begin{array}{cc}
0 & a_{-+}^{z}(t, \lambda) \\
a_{+-}(t, \lambda) & 0
\end{array}\right)
$$

where

$$
\left.\begin{array}{l}
a_{-+}^{z}(t, \lambda)=\frac{\sinh \left[\left(\theta^{z}(t)-\pi\right)\left(\lambda+i\left(\frac{1}{p}+\beta^{z}(t)\right)\right)\right]}{\sinh \pi\left(\lambda+i\left(\frac{1}{p}+\beta^{z}(t)\right)\right)} g_{-+}(t, t) \\
a_{+-}^{z}(t, \lambda)=\frac{\sinh \left[\left(\theta^{z}(t)-\pi\right)\left(\lambda+i\left(\frac{1}{p}+\beta^{z}(t)\right)\right)\right]}{\sinh \pi\left(\lambda+i\left(\frac{1}{p}+\beta^{z}(t)\right)\right)} g_{+-}(t, t) .
\end{array}\right\}
$$

Let us consider the operator

$$
B_{\Gamma} u(x)=a(x) u(x)+\left(A_{\Gamma} u\right)(x) \quad(x \in \Gamma)
$$

where the matrix function $a(x)$ has the components in $C(\Gamma \backslash F)$ and

$$
\left.\begin{array}{l}
a_{+}^{z}(t)=a\left(z+t \exp i \omega_{+}^{z}(t)\right) \\
a_{-}^{z}(t)=a\left(z-t \exp i \omega_{-}^{z}(t)\right)
\end{array}\right\}
$$

are matrix functions with components satisfying conditions (14) - (15) in a neighborhood of the point $t=0$. Set

$$
a^{z}(t)=\left(\begin{array}{cc}
a_{+}^{z}(t) & 0 \\
0 & a_{-}^{z}(t)
\end{array}\right) .
$$

Theorem 17. The operator $B_{\Gamma}: L_{p}^{n}(\Gamma, w) \rightarrow L_{p}^{n}(\Gamma, w)$ is locally invertible at the point $z \in F$ if and only if its local symbol $\sigma^{2}\left(B_{\Gamma}\right)(t, \lambda)=a^{z}(t)+\sigma^{z}(A)(t, \lambda)$ satisfies the condition

$$
\varliminf_{t \rightarrow 0} \inf _{\lambda \in \mathbb{R}}\left|\operatorname{det} \sigma^{2}\left(B_{\Gamma}\right)(t, \lambda)\right|>0 .
$$

Proof. The proof of this theorem follows from Proposition 15 and Theorem 6

As above, we say that $\varsigma \in \mathbb{C}$ is a point of the local spectrum of the operator $A_{\Gamma}$ at the point $x \in \Gamma$ if $A_{\Gamma}-\varsigma I$ is not locally invertible at the point $x$. We denote by $S p_{x} A_{\Gamma}$ the local spectrum of $A_{\Gamma}$ at the point $x$.

Corollary 18. Let $z \in F$. Then $\varsigma \in S p_{z} A_{\Gamma}$ if and only if there exists a sequence $t_{m} \rightarrow 0$ such that $\lim _{m \rightarrow \infty} \inf _{\lambda \in \mathbb{R}}\left|\operatorname{det} \sigma^{z}\left(A_{\Gamma}-\varsigma I\right)\left(t_{m}, \lambda\right)\right|=0$. Thus, the local spectrum of $A_{\Gamma}$ at the singular point $z$ is

$$
S p_{z} A_{\Gamma}=\bigcup\left\{\varsigma \in \mathbb{C}: \operatorname{det} \tilde{\sigma}^{z}\left(A_{\Gamma}-\varsigma I\right)(\lambda)=0 \text { for some } \lambda \in \mathbb{R} \cup\{\infty\}\right\}
$$

where $\operatorname{det} \tilde{\sigma}^{2}\left(A_{\Gamma}-\varsigma I\right)(\lambda)$ is the set of the partial limits of $\operatorname{det} \sigma^{2}\left(A_{\Gamma}-\varsigma I\right)(t, \lambda)$ as $t \rightarrow 0$.

One can see that the local spectrum of the potential operator $A_{r}: L_{p}^{n}(\Gamma, w) \rightarrow$ $L_{p}^{n}(\Gamma, w)$ may be a massive set, that is, a set with a positive planar Lebesgue measure. There are three reasons for $S p_{z} A_{\Gamma}$ to be a massive set:

1) the oscillation of the curve $\Gamma$

2) the oscillation of the coefficients

3 ) the oscillation of the weight at the singular point $z$. 
Theorem 19. The operator $B_{\Gamma}: L_{p}^{n}(\Gamma, w) \rightarrow L_{p}^{n}(\Gamma, w)$ is a Fredholm operator if and only if

1) $\inf _{x \in \Gamma}|\operatorname{det} a(x)|>0$

2) $\underline{\lim }_{t \rightarrow 0} \inf _{\lambda \in \mathbb{R}}\left|\operatorname{det} \sigma^{z}\left(B_{\Gamma}\right)(t, \lambda)\right|>0$ for any point $z \in F$.

If both conditions are fulfilled, then

$$
\text { Ind } B_{\Gamma}=-\sum_{z \in F} \frac{1}{2 \pi}\left[\arg \operatorname{det}\left(I+a^{z}\left(t_{0}\right)^{-1} \sigma^{z}\left(A_{\Gamma}\right)\left(t_{0}, \lambda\right)\right]_{\lambda=-\infty}^{\infty}\right.
$$

where $t_{0}$ is any point close enough to 0.

Proof. Simonenko's local principle [20] says that $B_{\Gamma}: L_{p}^{n}(\Gamma, w) \rightarrow L_{p}^{n}(\Gamma, w)$ is a Fredholm operator if and only if $B_{\Gamma}$ is locally invertible at every point $x \in \Gamma$. The operator $A_{\Gamma}$ is locally compact at any point $x \in \Gamma \backslash F$. The operator $B_{\Gamma}$ is locally equivalent (in the sense of [20]) to the operator $a(x) I$ at a point $x \in \Gamma \backslash F$. This implies that condition 1 ) is necessary and sufficient for the local invertibility of $B_{\Gamma}$ at non-singular points. From Theorem 17 it follows that condition 2) is necessary and sufficient for the local invertibility of $B_{\mathrm{r}}$ at singular points $z \in F$. The index formula can be proved, by the standard method of separation of singularities (see, for instance, $[18,19])$ and is based on formula (10)

Remark 2. The Fredholm spectrum of the operator $A_{\Gamma}$ is given by the formula ess $s p A_{\Gamma}=\cup_{z \in F} S p_{z} A_{\Gamma}$.

\section{Integral operator of the Dirichlet problem}

Let

$$
A_{\Gamma} u(x)=\frac{1}{\pi} \int_{\Gamma} \frac{(\nu(y), x-y)}{|x-y|^{2}} u(y) d l_{y} \quad(x \in \Gamma)
$$

be the double layer potential on a Jordan curve $\Gamma$ satisfying the conditions of the previous section. Moreover, suppose that the singular points $z \in F$ are points with zero rotation, that is, $\lim _{t \rightarrow 0} \delta^{z}(t)=0$ for all $z \in F$. We consider $A_{\Gamma}$ as an operator acting in $L_{p}(\Gamma, w)$ where the weight $w$ satisfies the above conditions. Let

$$
r_{z}\left(A_{\Gamma}\right)=\sup \left\{|\zeta|: \zeta \in \mathbb{C} \text { and } A_{\Gamma}-\zeta I \text { not locally invertible in } L_{p}(\Gamma, w)\right\}
$$

be the local spectral radius $A_{\Gamma}$.

Theorem 20. Let the above given conditions for the curve $\Gamma$ hold. Then

$$
r_{z}\left(A_{\Gamma}\right)=\varlimsup_{i \rightarrow 0} \frac{\sin \left|\pi-\theta^{z}(t)\right|\left(\frac{1}{p}+\beta^{z}(t)\right)}{\sin \pi\left(\frac{1}{p}+\beta^{z}(t)\right)} .
$$

Proof. We have

$$
\begin{aligned}
\sigma^{2}\left(A_{\Gamma}-\zeta I\right)(t, \lambda) & \\
\quad= & \left.\begin{array}{cc}
-\zeta & \sinh \left[\left(\theta^{2}(t)-\pi\right)\left(\lambda+i\left(\frac{1}{p}+\beta^{x}(t)\right)\right)\right] \\
\sinh \pi\left(\lambda+i\left(\frac{1}{p}+\beta^{2}(t)\right)\right) & -\zeta
\end{array}\right)
\end{aligned}
$$


From Theorem 17 we deduce that

$$
r_{z}\left(A_{\Gamma}\right)=\varlimsup_{\ell \rightarrow 0} \sup _{\lambda \in \mathbb{R}}\left|\frac{\sinh \left[\left(\theta^{z}(t)-\pi\right)\left(\lambda+i\left(\frac{1}{p}+\beta^{z}(t)\right)\right)\right]}{\sinh \pi\left(\lambda+i\left(\frac{1}{p}+\beta^{z}(t)\right)\right)}\right| .
$$

It is well-known (see, for instance, [5]) that

$$
\sup _{\lambda \in \mathbb{R}}\left|\frac{\sinh [\xi(\lambda+i \gamma]}{\sinh \pi(\lambda+i \gamma)}\right|=\frac{\sin \xi \gamma}{\sin \pi \gamma} \quad(0<\xi<\pi, \gamma \in(0,1)) .
$$

Hence formulas (31) and (32) yield (30)

Let us now consider the integral operator $B_{\Gamma}=I+A_{\Gamma}$ corresponding to the interior Dirichlet problem for harmonic functions.

Theorem 21. The operator $B_{\Gamma}: L_{p}(\Gamma, w) \rightarrow L_{p}(\Gamma, w)$ is locally invertible at a singular point $z \in F$ if and only if $\frac{1}{p} \notin\left[a_{-}^{z}, a_{+}^{z}\right]$ where

$$
\left.\begin{array}{l}
a_{-}^{z}=\underline{\lim }_{t \rightarrow 0}\left(\frac{\pi}{\pi+\left|\theta^{z}(t)-\pi\right|}-\beta^{z}(t)\right) \\
a_{+}^{z}=\varlimsup_{t \rightarrow 0}\left(\frac{\pi}{\pi+\left|\theta^{z}(t)-\pi\right|}-\beta^{z}(t)\right) .
\end{array}\right\}
$$

Proof. We have

$$
\operatorname{det} \sigma^{2}\left(B_{\Gamma}\right)(t, \lambda)=1-\left[\frac{\sinh \left[\left(\theta^{2}(t)-\pi\right)\left(\lambda+i\left(\frac{1}{p}+\beta^{z}(t)\right)\right)\right]}{\sinh \pi\left(\lambda+i\left(\frac{1}{p}+\beta^{z}(t)\right)\right)}\right]^{2}
$$

The equation $1-\frac{\sinh ^{2}|\pi-\theta| \zeta}{\sinh ^{2} \pi \zeta}=0 \quad(\zeta=\lambda+i \eta \in \mathbb{R}+i(0,1))$ has a unique solution $\zeta=i \frac{\pi}{\pi+|\theta-\pi|}$. Hence, if $\frac{1}{p} \notin\left[a_{-}^{z}, a_{+}^{z}\right]$, then condition (29) of Theorem 16 is fulfilled. This yields the local invertibility of $B_{\Gamma}$ at the point $z$. If $\frac{1}{p} \in\left[a_{-}^{z}, a_{+}^{z}\right]$, then there is a sequence $t_{m} \rightarrow 0$ such that $\lim _{m \rightarrow \infty} \frac{\pi}{\pi+\left|\theta^{2}\left(t_{m}\right)-\pi\right|}-\beta^{z}\left(t_{m}\right)=\frac{1}{p}$. Thus in this case condition (29) is not fulfilled, which shows that $B_{\Gamma}$ is not a locally invertible operator at the point $z$

Remark 3. The oscillations of the curve and the weight at singular points define a segment $\left[a_{-}^{z}, a_{+}^{z}\right]$ of prohibited values of $\frac{1}{p}$ for the local invertibility of the operator $B_{\Gamma}$. Note that in the case of an angular singular point and a power weight there is exactly one prohibited value of $\frac{1}{p}$.

Theorem 22. The operator $B_{\Gamma}: L_{p}(\Gamma, w) \rightarrow L_{p}(\Gamma, w)$ is of Fredholm type if and only if the condition of Theorem 21 is satisfied for all singular points $z \in F$. In this case

$$
\text { Ind } B_{\Gamma}=\sum_{z \in F} \kappa_{z}(p) \quad \text { where } \kappa_{z}(p)= \begin{cases}1 & \text { if } \frac{1}{p}>a_{+}^{z} \\ 0 & \text { if } \frac{1}{p}<a_{-}^{z} .\end{cases}
$$

Proof. The Fredholm criterion for $B_{\Gamma}$ follows from Simonenko's local principle [20] and Theorem 21. The function $\Delta(\zeta)=1-\left[\frac{\sinh [|\theta-\pi| \zeta \mid}{\sinh \pi(\zeta)}\right]^{2}(\zeta=\lambda+i \eta)$ is analytic in the 
strip $\operatorname{Im} \zeta \in(0,1)$, it extends continuously onto the real axis, we have $\lim _{\lambda \rightarrow \infty} \Delta(\lambda+$ $i \eta)=1$, and $\Delta(\zeta)$ has only one zero $\zeta=\frac{i \pi}{\pi+|\theta-\pi|}$ in this strip. It is easy to check that $[\arg \Delta(\lambda)]_{\lambda=-\infty}^{\infty}=0$ because $\Delta(\lambda)(\lambda \in \mathbb{R} \cup\{\infty\})$ is a real-valued function. Thus, the argument principle gives

$$
-\frac{1}{2 \pi}[\Delta(\lambda+i \eta)]_{\lambda=-\infty}^{\infty}= \begin{cases}1 & \text { if } \eta>\frac{\pi}{\pi+|\theta-\pi|} \\ 0 & \text { if } \eta<\frac{\pi}{\pi+|\theta-\pi|} .\end{cases}
$$

Now formulas (34) and (30) yield (33)

Acknowledgement. The author is very indebted to Prof. A. Böttcher who improved the English.

\section{References}

[1] Böttcher, A. and Yu. I. Karlovich: Carleson Curves, Muckenhoupt Weights, and Toeplitz Operators (Progress in Mathematics: Vol. 154). Basel - Boston - Berlin: Birkhäuser Verlag 1997.

[2] Radon, I.: On boundary value problems for logarithmic potential (in Russian). Uspehi Math. Nauk 1 (1946)3-4, 96 - 124.

[3] Lopatinskiy, Ya. B.: On a type of singular integral equations (in Ukrainian). Theor. Appl. Math. Lvov State Univ. (1963)2, 53 - 57.

[4] Daniluk, I. I.: Nonregular Boundary Value Problems on the Plain (in Russian). Moscow: Nauka 1975.

[5] Shelepov, V. Yu.: On index and spectrum of integral operators of potential type along the Radon curves (in Russian). Math. sbornik 181 (1990), 751 - 778.

[6] Prössdorf, S.: Linear integral equations (in Russian). Itogi Nauki i Techniki. Sovremennie Problemi Matematiki. Fundamentalnie Napravlenia 27 (1988), 5 - 130.

[7] Maz'ya, V. G.: Boundary integral equations (in Russian). Itogi Nauki i Techniki. Sovremennie Problemi Matematiki. Fundamentalnie Napravlenia 27 (1988), 131 - 237.

[8] Maz'ya, V. and A. Solov'ev: On the boundary integral equations of the Dirichlet problems in a plane domains with peaks at the boundary (in Russian). Mat. sbornik 180 (1989), 1211 - 1223; English transl. in: Math. USSR Sbornik 68 (1991), 61 - 83.

[9] Maz'ya, V. and A. Solov'ev: On the boundary integral equations of the logarithmic potential theory for domains with peaks. Rend. Mat. Acc. Linceei (Ser. 9) 6 (1995), $211-236$.

[10] Maz'ya, V. and A. Solov'ev: Boundary integral equations on a cuspidal contour. Appl. Anal. 65 (1997), 289 - 305.

[11] Gohberg, I. and M. G. Krein: Systems of integral equations on half-line with kernels depending on a difference of arguments (in Russian). Uspehi Matem. Nauk. 13 (1958)2, $3-72$.

[12] Rabinovich, V. S.: Singular integral operators on a composed contour with oscillating tangent and pseudodifferential Mellin operators. Soviet Math. Dokl. 44 (1992), $791-796$.

[13] Rabinovich, V.S.: Singular integral operators on composed contours and pseudodifferential operators (in Russian). Matem. Zametki 58 (1995), $65-85$. 
[14] Rabinovich, V. S.: Mellin pseudodifferential operators techniques in the theory of singular. integral operators on some Carleson curves. Operator Theory: Advances and Applications 102 (1998), $201-218$.

[15] Böttcher, A., Karlovich, Yu. I. and V. S. Rabinovich: Emergence, persistence, and disappearance of logarithmic spirals in the spectra of singular integral operators. Int. Equ. Oper. Theory 25 (1996), $406-444$.

[16] Böttcher, A., Karlovich, Yu. I. and V. S. Rabinovich: Mellin pseudodifferential operators with slowly varying symbols and singular integrals on Carleson curves with Muckenhoupt weights. Man. Math. 95 (1998), 363 - 376.

[17] Rabinovich, V. S.: Pseudodifferential operators with analytic symbols and some of their applications. In: Proc. Sem. Lin. Top. Spaces and Compl. Anal. (eds.: A. Aytuna). Ankara: Metu-Tübitak 1995, pp. 79 - 98.

[18] Böttcher, A. and B. Silbermann: Analysis of Toeplitz Operators. Berlin: Akademie-Verlag 1989 and Berlin - Heidelberg - New York: Springer-Verlag 1990.

[19] Gohberg, I. and N. Krupnik: One-Dimensional Linear Singular Integral Equations. Vols. I and II. Basel - Boston - Berlin: Birkhäuser Verlag 1992.

[20] Simonenko, I. B. and Chin Ngok Min: The Local Method in the Theory of One-Dimensional Singular Integral Equations with Pieceurise Continuous Coefficients. Noethericity (in Russian). Rostov (Russia): Univ. Press 1986.

Received 16.02.1999 